\title{
Perirenal hematoma in a patient treated with bevacizumab for metastatic colon cancer: A case report
}

\author{
MIN SUNG LEE，IL SANG SHIN，DO HYUNG KWUN, SE HYUNG KIM，HYUN JUNG KIM, \\ CHAN KYU KIM, SEONG KYU PARK, DAE SIK HONG and JINA YUN
}

Division of Hematology-Oncology, Department of Internal Medicine, Soonchunhyang University School of Medicine, Bucheon Hospital, Bucheon, Gyeonggi 420-767, Republic of Korea

Received February 21, 2015; Accepted February 16, 2016

DOI: $10.3892 / \mathrm{ol} .2016 .4328$

\begin{abstract}
The present study reports the case of a patient that developed spontaneous perirenal hematoma during treatment with bevacizumab-containing chemotherapy. A 44-year-old woman with metastatic sigmoid colon cancer, who was being treated with bevacizumab $(5 \mathrm{mg} / \mathrm{kg}$, intravenous, $90 \mathrm{~min}$ biweekly), was admitted to hospital following 3 cycles of chemotherapy, with a sudden onset of dyspnea and oliguria. An emergency hemodialysis was performed and a large right perirenal hematoma was diagnosed using computed tomography. The patient was immediately instructed to discontinue chemotherapy, including bevacizumab. However, the right perirenal hematoma increased in size and a left perirenal hematoma developed 3 weeks later. The two perirenal hematomas stabilized 7 weeks subsequent to the termination of bevacizumab treatment. Spontaneous perirenal hematoma due to bevacizumab treatment is an extremely rare occurrence. However, physicians should be aware of this potential complication associated with bevacizumab treatment.
\end{abstract}

\section{Introduction}

Bevacizumab is a recombinant humanized monoclonal antibody that prevents tumor angiogenesis by inhibiting vascular endothelial growth factor (VEGF) (1). Bevacizumab is widely used in the treatment of a variety of advanced solid tumors, including colorectal cancer (CRC), non-small cell lung cancer (NSCLC), breast cancer and renal cell cancer (RCC).

The adverse-effect profile of bevacizumab is different compared with traditional cytotoxic chemotherapy agents (2). Bone marrow suppression and gastrointestinal (GI) toxicity are commonly associated with chemotherapy; by contrast,

Correspondence to: Dr Jina Yun,Division of Hematology-Oncology, Department of Internal Medicine, Soonchunhyang University School of Medicine, Bucheon Hospital, 1174 Jung-Dong, Bucheon, Gyeonggi 420-767, Republic of Korea

E-mail: 19983233@schmc.ac.kr; 19983233@naver.com

Key words: hematoma, bevacizumab, colonic neoplasms frequently reported adverse effects of bevacizumab include hypertension, proteinuria and epistaxis (2). Bevacizumab is also associated with other serious side effects, such as hemorrhage, GI tract perforation, wound dehiscence, arterial and venous thromboembolism, hypertensive crisis, reversible posterior leukoencephalopathy, neutropenia, infections, nephritic syndrome and congestive heart failure (3). The importance of bevacizumab-associated hemorrhage is highlighted by the Food and Drug Administration (FDA)-issued black box warning, which recognizes that in patients with NSCLC treated with bevacizumab-containing chemotherapy, fatal pulmonary hemorrhage may occur. However, little is known regarding spontaneous perirenal hematoma associated with bevacizumab treatment. To date, only one case has been reported in the literature (4). The patient was treated with bevacizumab $(5 \mathrm{mg} / \mathrm{kg}$, intravenous, 90 min biweekly), but developed perirenal hematoma after 11 cycles of chemotherapy (4). The patient received a conservative treatment approach and experienced symptomatic improvement (4).

The present study reports the case of a 44-year-old woman that developed a perirenal hematoma during treatment with bevacizumab-containing chemotherapy for colon cancer.

\section{Case report}

In April 2012, a 44-year-old woman presented at Soonchunhyang University Bucheon Hospital (Bucheon, Korea) emergency room suffering with hematochezia and abdominal pain for 10 days. Contrast-enhanced computed tomography (CT) and colonoscopy revealed a diffuse wall thickening on the proximal sigmoid colon with metastasis to the lungs, peritoneum, T2 vertebrae, retroperitoneum and hilar, and subcarinal lymph nodes. The patient was diagnosed with sigmoid colon cancer [poorly-differentiated adenocarcinoma; stage IV, pT3N2M1 (5); epidermal growth factor receptor $3^{+} /$Kirsten ras (KRAS)-wild-type] with metastasis to the lungs, peritoneum, T2 vertebrae, retroperitoneum and hilar and subcarinal lymph nodes. The patient underwent a palliative anterior resection for a partial colon obstruction. Prior to the initiation of chemotherapy, baseline CT scans were obtained 4 weeks subsequent to surgery and no evidence of perirenal hematoma was observed (Fig. 1). Palliative chemotherapy consisting of the oxaliplatin $\left(85 \mathrm{mg} / \mathrm{m}^{2}\right.$, intravenous, 


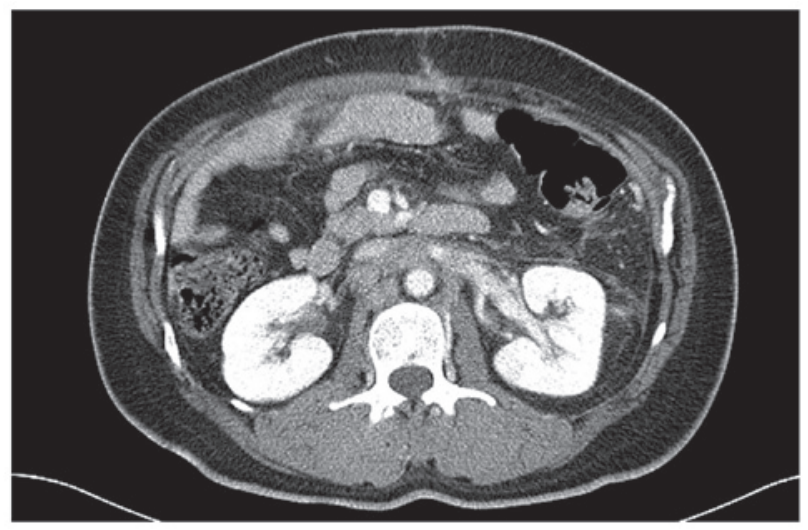

Figure 1. A baseline computed tomography scan revealing no evidence of a perirenal hematoma.

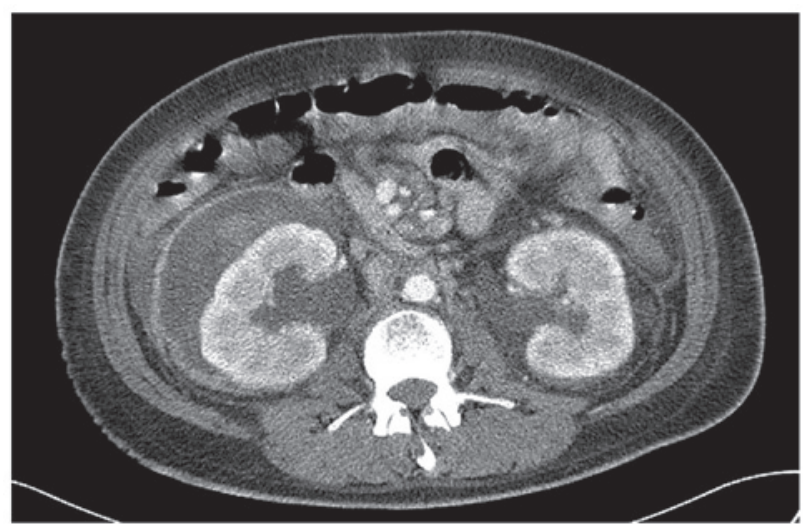

Figure 2. An abdominal computed tomography scan subsequent to hemodialysis revealing a large right perirenal hematoma with no active bleeding.

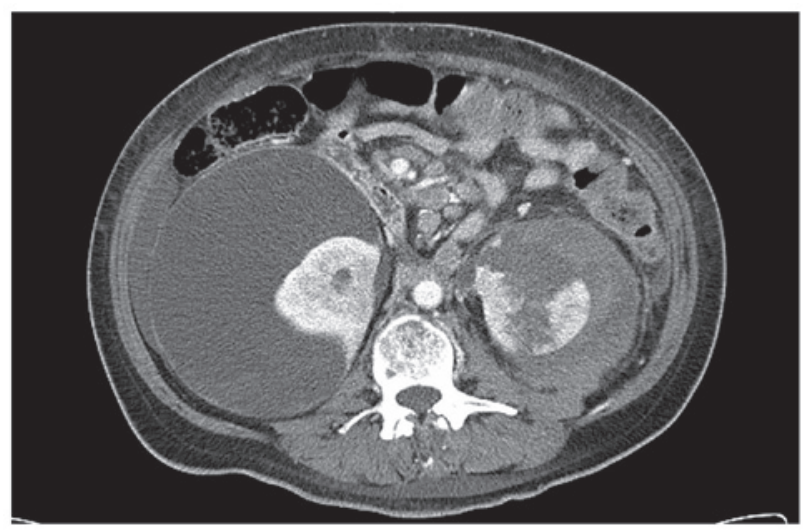

Figure 3. A follow-up computed tomography scan 3 weeks subsequent to hemodialysis revealing that the right perirenal hematoma had increased in size, and that a left perirenal hematoma with renal parenchyma disruption and laceration had developed.

$120 \mathrm{~min}$ biweekly), leucovorin $\left(400 \mathrm{mg} / \mathrm{m}^{2}\right.$, intravenous, $120 \mathrm{~min}$ biweekly) and 5-fluorouracil $\left(2,400 \mathrm{mg} / \mathrm{m}^{2}\right.$, intravenous, 44 h biweekly) (FOLFOX6) regimen was administered, and bevacizumab ( $5 \mathrm{mg} / \mathrm{kg}$ every 2 weeks) was added to the treatment regimen following the first cycle of FOLFOX6. At the fourth cycle of chemotherapy (June 2015), the patient presented with dyspnea and oliguria. The chemotherapy

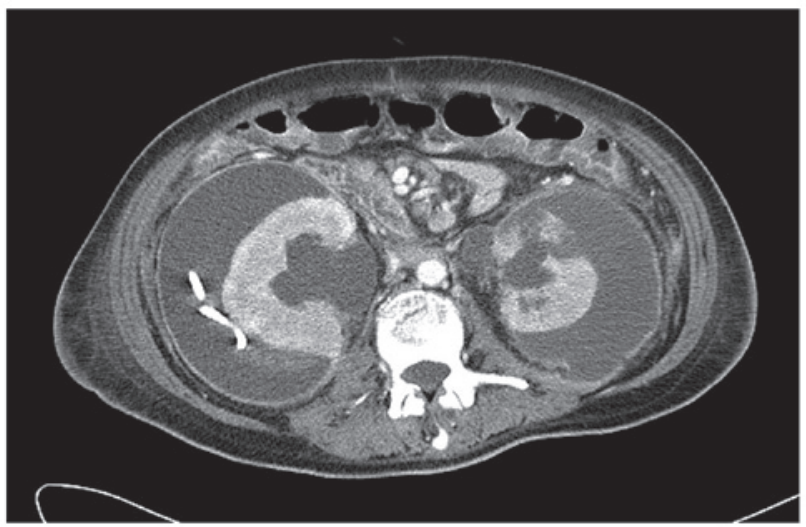

Figure 4. A follow-up computed tomography scan 7 weeks subsequent to the termination of bevacizumab treatment revealing persistent perirenal hematoma on the right and left kidneys with no evidence of active bleeding.

cycle was delayed, and laboratory tests were performed to identify the cause of the symptoms. Blood test results revealed that the patient had an elevated level of white blood cells $\left(10.86 \times 10^{9}\right.$ cells/1; normal range, $4-10 \times 10^{9}$ cells $\left./ 1\right)$ and C-reactive protein (49 $\mathrm{mg} / 1$; normal range, $0-5 \mathrm{mg} / \mathrm{l}$ ), suggesting an underlying inflammatory condition. In addition, the patient had a serum urea nitrogen level of $17.8 \mathrm{mmol} / 1$ (normal range, 2.9-7.1 nmol/l), creatinine level of $530.4 \mu \mathrm{mol} / 1$ (normal range, $53-115 \mu \mathrm{mol} / \mathrm{l}$ ) and potassium level of $5.9 \mathrm{mmol} / 1$ (normal range, $3.5-5.5 \mathrm{nmol} / \mathrm{l}$ ). Sodium (133 mmol/l; normal range, 133-142 mmol/l) and chloride (99 mmol/1; normal range, 98-110 mmol/1) levels were normal, and the patient exhibited no signs of proteinuria. An arterial blood gas analysis revealed compensated metabolic acidosis with hypoxia. Chest radiography revealed that the patient had pulmonary congestion and pleural effusion. Consequently, emergency hemodialysis was initiated.

Following hemodialysis, the general condition of the patient improved. A CT scan revealed the presence of a large right perirenal hematoma, $11.2 \times 9.7 \mathrm{~cm}$ in size, with no evidence of active bleeding (Fig. 2). The hematoma contained fluid and high-density amorphous materials. The patient was treated conservatively, including bed rest, a blood transfusion with 6 units of packed red blood cells, hemodialysis and prophylactic antibiotic therapy with close monitoring. A follow-up CT scan 3 weeks subsequent to hemodialysis revealed that the right perirenal hematoma had increased in size and a left perirenal hematoma with renal parenchyma disruption and laceration had developed (Fig. 3). The patient underwent ultrasonography-guided percutaneous catheter drainage for the right perirenal hematoma. However, intervention on the left side was terminated, due to the patient exhibiting unstable vital signs [systolic blood pressure, $192 \mathrm{mmHg}$ (normal range $<120 \mathrm{mmHg}$ ); heart rate, 130 beats/min (normal range, 60-100 beats/min); blood oxygen saturation, 79\% (normal range, 95-100\%)]. An additional CT scan was performed 4 weeks subsequent to the previous CT scan, 7 weeks subsequent to the termination of bevacizumab treatment, which demonstrated that neither of the perirenal hematomas had progressed in size (Fig. 4). The patient received the best supportive care, but succumbed 4 weeks later to the rapidly progressing cancer. 


\section{Discussion}

Perirenal hematomas commonly arise from a traumatic renal injury (4). However, spontaneous perirenal hematomas are rare (4). In a meta-analysis of 47 publications and 165 cases in 2002, Zhang et al (6) analyzed the etiology of spontaneous perirenal hematomas. The most common etiology was malignancy, including renal cell carcinoma in $33.3 \%$ of cases, followed by a non-malignant tumor, including angiomyolipoma in $24.4 \%$ of the cases, an arteriovenous malformation in $17.9 \%$ of the cases, an infection in $10.3 \%$, of the cases, nephritis in $5.1 \%$ of the cases and hematological disease in $5.1 \%$ of the cases. There are a few cases of perirenal hematomas that are idiopathic (7). Among dialysis patients, spontaneous perirenal hematomas have been infrequently reported in the literature. It is possible that hemorrhage may occur as a result of acquired cystic change (8). The present patient had no pathological renal conditions, including renal primary cancer, renal metastasis, vascular diseases and acquired cystic kidney disease.

Bevacizumab was the first anti-angiogenic agent approved for use in cancer by the USA FDA in 2004 (9). Angiogenesis inhibitors, in particular inhibitors of the VEGF pathway, have been associated with significant bleeding complications (9). In a meta-analysis of 20 randomized controlled trials, which consisted of 12,617 patients with a variety of solid tumors, bevacizumab was identified to significantly increase the risk of bleeding, with a relative risk (RR) of 2.48 [95\% confidence interval (CI), 1.93-3.18] compared with control groups (10). However, among the various bleeding complications associated with bevacizumab treatment, perirenal hematoma has only been previously reported by one study. Hayashi et al (4) reported a case of a 59-year-old woman with metastatic rectal cancer treated with bevacizumab, who developed lower-back pain following 11 cycles of chemotherapy. A CT scan revealed the presence of a perirenal hematoma, and the discontinuation of bevacizumab treatment resulted in an improvement of symptoms.

Consequently, there have been only two reported cases of perirenal hematoma in patients undergoing bevacizumab treatment, including the present case. In the two studies, the patients were diagnosed with metastatic colon cancer. The present study hypothesizes that the occurrence of spontaneous perirenal hematoma associated with bevacizumab treatment, which is a rare event, may be due to the high relative risk of bleeding in colorectal cancer. Patients with other tumors may be at a different risk of bleeding, due to variations in tumor biology and treatments. In a previous meta-analysis, the risk of high-grade bleeding associated with bevacizumab treatment was relatively increased in patients with NSCLC (RR, 3.41; 95\% CI, 1.68-6.91), RCC (RR, 6.37; 95\% CI, 1.43-28.33) and CRC (RR, 9.11; 95\% CI, 1.70-48.79) compared with metastatic breast cancer (RR, 1.33; 95\% CI, 0.32-5.46) and metastatic pancreatic cancer (RR, 1.54; 95\% CI, 0.51-4.64) (11).

The most common symptom of perirenal hematoma is flank pain (6). However, the initial presenting symptom of the present patient was oliguria. Therefore, the cause of the acute renal failure (ARF) of the present patient was unclear. In total, 2 weeks prior to presentation, the creatinine levels of the patient were normal, but then abruptly increased. Perirenal hematoma is known to cause $\operatorname{ARF}(12,13)$. There was little evidence to suspect any other cause for the sudden elevation in creatinine levels.
Therefore, the present study concluded that the massive perirenal hematoma likely contributed to the ARF in the present study.

Notably, in the present study, following the discontinuation of bevacizumab treatment, the perirenal hematoma increased in size and a novel perirenal hematoma developed. According to pharmacokinetic studies of bevacizumab, the half-life of bevacizumab is relatively long; the mean half-life of bevacizumab is $\sim 21$ days, but the range varies between 11 and 50 days (14). Considering that the two perirenal hematomas observed in the present patient did not stabilize until 7 weeks subsequent to the cessation of treatment, the present study hypothesizes that the residual circulating bevacizumab aggravated the perirenal hematomas up to 7 weeks following the cessation of treatment.

Overall, the present study concludes that bevacizumab is likely to have contributed to the development of the perirenal hematoma. Although the development of a spontaneous perirenal hematoma caused by bevacizumab treatment is an extremely rare event, it may lead to serious complications. Furthermore, due to the perirenal hematoma and ARF, the patient in the present study could not receive any additional chemotherapy and the patient succumbed to uncontrolled colon cancer.

In conclusion, patients undergoing bevacizumab treatment for cancer should be closely monitored for bleeding complications. In particular, when colorectal cancer patients receive bevacizumab-containing chemotherapy, physicians should be aware of unusual bleeding complications, including perirenal hematomas.

\section{References}

1. Ranieri G, Patruno R and Ruggieri E: Vascular endothelial growth factor (VEGF) as a target of bevacizumab in cancer: from the biology to the clinic. Curr Med Chem 13: 1845-1857, 2006.

2. Hurwitz H and Saini S: Bevacizumab in the treatment of metastatic colorectal cancer: Safety profile and management of adverse events. Semin Oncol 33 (Suppl 10): S26-S34, 2006.

3. Saif MW: Managing bevacizumab-related toxicities in patients with colorectal cancer. J Support Oncol 7: 245-251, 2009.

4. Hayashi H, Okamoto I and Nakagawa K: Perirenal hematoma associated with bevacizumab treatment. Invest New Drugs 30: 808-809, 2012.

5. Edge SB, Byrd DR, Compton CC, Fritz AG, Greene FL and Trotti A (eds.). AJCC Cancer Staging Manual. 7th edition. Springer, New York, NY, 2010.

6. Zhang JQ, Fielding JR and Zou KH: Etiology of spontaneous perirenal hemorrhage: A meta-analysis. J Urol 167: 1593-1596, 2002.

7. McDougal WS, Kursh ED and Persky L: Spontaneous rupture of the kidney with perirenal hematoma. J Urol 114: 181-184, 1975.

8. Milard PR and Oliver D: Acquired cystic disease of the kidneys: a hazard of long-term intermittent maintenance haemodialysis. J Clin Pathol 30: 868-877, 1977.

9. Folkman J: Angiogenesis: An organizing principle for drug discovery? Nat Rev Drug Discov 6: 273-286, 2007.

10. Hapani S, Sher A, Chu D and Wu S: Increased risk of serious hemorrhage with bevacizumab in cancer patients: A meta-analysis. Oncology 79: 27-38, 2010.

11. Hang XF, Xu WS, Wang JX, et al: Risk of high-grade bleeding in patients with cancer treated with bevacizumab: A meta-analysis of randomized controlled trials. Eur J Clin Pharmacol 67: 613-623, 2011.

12. Gültekin N, Akın F and Küçükateş E: Warfarin-induced bilateral renal hematoma causing acute renal failure. Turk Kardiyol Dern Ars 39: 228-230, 2011.

13. Singh N, Neubauer BE and Venuto RC: Acute renal failure during pregnancy secondary to spontaneous perirenal hematoma. Ren Fail 29: 1053-1054, 2007.

14. Gordon MS, Margolin K, Talpaz M, Sledge GW Jr, Holmgren E, Benjamin R, Stalter S, Shak S and Adelman D: Phase I safety and pharmacokinetic study of recombinant human anti-vascular endothelial growth factor in patients with advanced cancer. J Clin Oncol 19: 843-850, 2001. 